\title{
Article \\ Nucleosynthesis, Mixing Processes, and Gas Pollution from AGB Stars
}

\author{
Paolo Ventura ${ }^{1, *(D)}$, Flavia Dell'Agli ${ }^{1}$, Marco Tailo ${ }^{2}$, Marco Castellani ${ }^{1}$, Ester Marini ${ }^{1,3,4,5}$ and Silvia Tosi ${ }^{6}$ \\ and Marcella Di Criscienzo ${ }^{1}$
}

1 INAF Osservatorio Astronomico di Roma, Via Frascati 33, 00078 Monte Porzio Catone, Italy; flavia.dellagli@inaf.it (F.D.); marco.castellani@inaf.it (M.C.); ester.marini@inaf.it (E.M.); marcella.dicriscienzo@inaf.it (M.D.C.)

2 Dipartimento di Fisica e Astronomia Augusto Righi, Universitá degli Studi di Bologna, Via Gobetti 93/2, 40129 Bologna, Italy; mrctailo@gmail.com

3 Nordita, KTH Royal Institute of Technology and Stockholm University, Hannes Alfvens väg 12, 11421 Stockholm, Sweden

4 Instituto de Astrofisica de Canarias (IAC), E-38200 La Laguna, Spain

5 Departamento de Astrofisica, Universidad de La Laguna (ULL), E-38206 La Laguna, Spain

6 Dipartimento di Matematica e Fisica, Universitá degli Studi Roma Tre, Via della Vasca Navale 84, 00100 Rome, Italy; silviatosi93@gmail.com

* Correspondence: paolo.ventura@inaf.it

check for updates

Citation: Ventura, P.; Dell'Agli, F.; Tailo, M.; Castellani, M.; Marini, E.; Tosi, S.; Di Criscienzo, M.

Nucleosynthesis, Mixing Processes, and Gas Pollution from AGB Stars. Universe 2022, 8, 45. https:/ / doi.org/10.3390/universe8010045

Academic Editor: Sara Palmerini

Received: 6 December 2021

Accepted: 5 January 2022

Published: 11 January 2022

Publisher's Note: MDPI stays neutral with regard to jurisdictional claims in published maps and institutional affiliations.

Copyright: (c) 2022 by the authors. Licensee MDPI, Basel, Switzerland. This article is an open access article distributed under the terms and conditions of the Creative Commons Attribution (CC BY) license (https:/ / creativecommons.org/licenses/by/ $4.0 /)$.

\begin{abstract}
We discuss the evolution of stars through the asymptotic giant branch, focusing on the physical mechanisms potentially able to alter the surface chemical composition and on how changes in the chemistry of the external regions affect the physical properties of the star and the duration of this evolutionary phase. We focus on the differences between the evolution of low-mass stars, driven by the growth of the core mass and by the surface carbon enrichment, and that of their higher mass counterparts, which experience hot bottom burning. In the latter sources, the variation of the surface chemical composition reflects the equilibria of the proton capture nucleosynthesis experienced at the base of the convective envelope. The pollution expected from this class of stars is discussed, outlining the role of mass and metallicity on the chemical composition of the ejecta. To this aim, we considered evolutionary models of $0.7-8 \mathrm{M}_{\odot}$ stars in a wide range of metallicities, extending from the ultra-metal-poor domain to super-solar chemistries.
\end{abstract}

Keywords: AGB and post-AGB; evolution; abundances

\section{Introduction}

The last decades have witnessed a growing interest towards the properties of stars evolving across the asymptotic giant branch (AGB). This is primarily due to the important role that these sources play in different astrophysical contexts, particularly as polluters of the interstellar medium. Indeed, during the AGB lifetime, they eject into the surrounding environment gas, which on the chemical side is significantly altered with respect to the initial chemistry, and dust, formed in the cool and expanded circumstellar envelopes [1]. It is for these reasons that AGB stars are believed to be extremely important to understand the chemical evolution of the Milky Way [2,3], the chemical patterns in star-forming regions of Local Group galaxies [4], and the formation of multiple populations in Globular Clusters [5].

AGB stars are extremely complex systems, composed by regions characterised by considerably different physical properties, ranging from the compact core, supported by the pressure of degenerate electrons, to the tenuous, extended envelope, which is gradually lost via stellar winds. These stars are supported on the energy side by the CNO nucleosynthesis taking place in an internal region above the CO core. Periodically, a helium-rich layer is ignited, causing a temporary extinction of the $\mathrm{CNO}$ activity and the drop in the overall 
energy production in the star: as these episodes take place under conditions of thermal instability [6], they are commonly referred to as "thermal pulses" (TP).

The alteration of the surface chemistry of AGB stars is due to different physical mechanisms, primarily connected to the nucleosynthesis activated in internal regions of the star, and to the efficiency of the mixing processes, which make the products of this nucleosynthesis reach the surface regions [7]. Unfortunately, the description of the AGB phase is made uncertain by the poor knowledge of some physical mechanisms from first principles, mainly the treatment of the convective instability [8] and of mass loss. The study of AGB stars is currently based on ad hoc, semi-empirical descriptions of these phenomena, which in some cases demand the tuning of free parameters.

The evolutionary properties of the stars evolving along the AGB exhibit an outstanding diversity, being extremely sensitive to the initial mass and metallicity. The former is relevant in assessing the relative importance of the various mechanisms able to alter the surface chemical composition, whereas metallicity affects the number of thermal pulses experienced by the star and the temperature at which the internal proton capture nucleosynthesis takes place, which in turn affects the equilibrium among the abundances of the different species.

In the present contribution, we present the main results of an ongoing research project developed by our research team during the last decade, aimed at describing the main aspects characterising the evolution of AGB stars, focusing on the different nucleosynthesis channels taking place in the interior and on the efficiency of the mixing processes. The role of the initial mass and of the chemical composition of the stars is widely discussed. Particular care is devoted to discussing the chemical composition of the ejecta of AGB stars of different masses and chemical compositions, to understand the role played by this class of stars as pollutants of the interstellar medium.

\section{The ATON Code for Stellar Evolution}

The results presented in this contribution were obtained by means of the ATON code for stellar evolution, which was described in detail in [9], where the interested reader can find an accurate analysis of the numerical structure and of the input physics adopted. Here, we recall the physical ingredients most relevant for the description of mixing during the advanced evolutionary phases and of the AGB phase itself.

Nuclear burning and mixing of chemicals are self-consistently coupled by means of a diffusive scheme, following the prescription by [10]. The temperature gradient with regions unstable to convective motions is found via the full spectrum of the turbulence model [11]. The rate of mass loss during the phases when the star is oxygen-rich at the surface is modelled via the treatment of [12]; for the carbon star phases, we used the results on mass loss published from the Berlin group $[13,14]$. The surface molecular opacities were calculated via the AESOPUS tool [15], which allows considering the effects of the variation of the surface abundances of the $\mathrm{CNO}$ species.

A detailed comparison between the results obtained by adopting these assumptions and those from other research groups have been so far presented for the solar [16] and sub-solar $[17,18]$ metallicities, for extremely metal-poor chemical compositions [19], and for super-solar chemistries [20]. These studies showed a substantial agreement on the physical and chemical properties characterising the evolution of low-mass (initial mass below $\sim 3 \mathrm{M}_{\odot}$, this threshold partly dependent on the metallicity investigated) stars, whereas significant differences are found in the massive AGB stars domain, mostly related to the differences in the treatment of the convective instability (this issue was analysed in detail by [8]).

In summary, in the first approximation, there is a general consensus among different research teams, regarding the results on the properties of low-mass AGB stars, which will be presented in the next sections. Conversely, the description of massive AGBs is extremely sensitive to the treatment of convection and mass loss, both mechanisms still poorly known from first principles: the results obtained are therefore sensitive to the input physics adopted. 


\section{Changes in the Surface Chemistry of Stars During the Red Giant Branch Phase}

Before entering the discussion on the AGB evolution of stars, we believe it important to focus on the changes in the surface chemical composition that take place during the RGB ascending, after the star has completed core hydrogen burning. This is because in low-mass stars, the changes occurring during the RGB in the surface abundance of some chemical species are comparable, or in some cases even higher than those experienced during the AGB phase.

After hydrogen is exhausted in the core, the stars expand as the burning region moves to a shell laying above the helium core. The general expansion and cooling of the external regions of the star favour the formation of a convective envelope, which grows bigger and bigger in mass, until reaching regions that have experienced some nuclear processing, with the result that the products of $\mathrm{H}$ burning are mixed to the surface. This is the so called "first dredge-up" (FDU) event, which represents the first convective episode during the life of stars able to alter the surface chemical composition.

The material mixed to the envelope by the FDU has been subjected to partial $\mathrm{H}$ burning, which means it is still mostly hydrogen, but with some added ${ }^{4} \mathrm{He}$ and the products of $\mathrm{CN}$ cycling. Typical surface abundance changes from FDU are an increase in the ${ }^{4} \mathrm{He}$ mass fraction by $\delta \mathrm{Y} \sim 0.03$, a decrease in the ${ }^{12} \mathrm{C}$ abundance by about $30 \%$, and an increase in the ${ }^{14} \mathrm{~N}$ and ${ }^{13} \mathrm{C}$ abundances. The surface ${ }^{12} \mathrm{C} /{ }^{13} \mathrm{C}$ ratio is expected to decrease from the initial value, slightly below 100, to 15-30. The effect of the FDU on other elements is relatively minor, although there is some dispute about sodium.

Standard models do not predict any further changes on the RGB once the FDU is complete. However, something must be occurring in real stars that is not predicted by the models, as some tension exists between the aforementioned theoretical predictions and results from the observations. For example, observations of the ${ }^{12} \mathrm{C} /{ }^{13} \mathrm{C}$ ratio in open metal-rich clusters reveal values in the 10-20 range [21,22], well below the predicted values of $\sim 25-30$. The deviation between theory and observations is even more striking in metal-poor field stars and in giants in GCs [23-25]. In some GCs, we see a clear decrease in $[\mathrm{C} / \mathrm{Fe}]$ with increasing luminosity on the RGB $[26,27]$, thus suggesting the action of some form of mixing connecting the hot region at the top of the H-burning shell with the convective envelope.

Observations generally indicate that the conflict with theory does not arise until the star has reached the luminosity that corresponds approximately to the point where the $\mathrm{H}$ shell has reached the discontinuity left behind by the FDU. The common understanding is that deep mixing begins once the advancing $\mathrm{H}$ shell removes the abundance discontinuity left behind by the FDU. This is connected with the inhibition of mixing associated with gradients in the chemical composition, so that mixing is free to develop only once they are removed [28].

The first possibility explored to solve this issue was that rotation, via meridional circulation currents, might be the reason for the large deep mixing occurring after the H-burning shell has crossed the discontinuity left behind by the FDU $[29,30]$. However, Reference [31] found that the best rotating models did not produce enough mixing to explain the decrease seen in the ${ }^{12} \mathrm{C} /{ }^{13} \mathrm{C}$ ratio on the upper RGB: the current belief is that rotating models do not reproduce the observations of RGB stars.

A further mechanism potentially able to favour deep mixing is thermohaline mixing. It was first suggested by [32], who found that a $\mu$ inversion, associated with the ignition of the ${ }^{3} \mathrm{He}+{ }^{3} \mathrm{He} \rightarrow{ }^{4} \mathrm{He}+2 \mathrm{p}$ reaction, develops naturally during the evolution along the RGB, when the $\mathrm{H}$ shell approaches the abundance discontinuity. This should initiate mixing just as the $\mathrm{H}$ shell approaches the discontinuity in the composition left behind by the FDU, in agreement with the observations. Unfortunately, the results are extremely sensitive to the free parameter $C$ entering the description of the thermohaline instability, although it is somewhat gratifying that so many observational constraints are matched by a value of $C \sim 1000$. Even if we assume that the mechanism identified by [32] is the one driving extra mixing, it is unsatisfactory having an idealised, yet approximate, theory that 
still contains a free parameter. We need a detailed hydrodynamical understanding of the process before firm conclusions can be drawn on this argument.

Whatever is the nature and the extent of the mixing besides the FDU experienced by the stars during the RGB ascending, it is important to understand in which cases the signature of this physical mechanism will affect the chemical composition of the star during the AGB phase. We focus on the species involved in $\mathrm{CN}$ cycling, as the other chemical elements are not affected by RGB mixing, with the only possible exception of sodium.

The effects of RGB mixing are erased during the AGB evolution of $M \geq 3 \mathrm{M}_{\odot}$ stars, as we will see that the surface chemistry will reflect the equilibria of the advanced proton capture nucleosynthesis activated at the base of the external convective region. On the other hand, the surface abundance of nitrogen in low-mass $\mathrm{M}<3 \mathrm{M}_{\odot}$ stars is almost entirely determined by the efficiency of the aforementioned mixing events taking place during the RGB ascending, as no significant further synthesis of ${ }^{14} \mathrm{~N}$ is expected during the AGB evolution of these stars. As regards carbon, we will see that the effects of RGB mixing will be relevant only in $\mathrm{M}<1-1.5 \mathrm{M}_{\odot}$ stars (the exact value depending on the metallicity), whose surface chemical composition is substantially unchanged during the AGB evolution.

\section{Chemical and Physical Process in AGB Stars: A General Overview}

The evolution of stars across the AGB offers a wide diversity and heterogeneity, both as concerns the evolution of the physical properties and the changes in the surface chemistry. The main aspects of the AGB evolution has been treated in different reviews, among which we mention the works by $[7,33,34]$.

On general grounds, AGB stars can be broadly divided into three groups, according to the relative importance of the physical mechanisms able to alter the surface chemical composition: low-mass stars, in which the surface chemical composition is substantially unchanged during the AGB phase; stars that experience carbon enrichment in the surface regions and may evolve as carbon stars; massive AGB stars, which experience hot bottom burning.

\subsection{Low-Mass O-Rich AGB Stars}

We first focus on stars of mass in the $0.7-1 \mathrm{M}_{\odot}$ range $^{1}$, which are composed by a $\sim 0.5 \mathrm{M}_{\odot}$ core and a $\sim 0.2-0.5 \mathrm{M}_{\odot}$ convective envelope. An example of the evolution of the main physical properties of such a star is shown in Figure 1, with the different panels reporting the change with the AGB time of the core and total mass, luminosity, and effective temperature. The results of Figure 1 refer to a $0.9 \mathrm{M}_{\odot}$ star with metallicity $\mathrm{Z}=8 \times 10^{-3}$, which is typical of stars in the Large Magellanic Cloud [35]. The stars in this mass domain suffer significant mass loss during the red giant branch (RGB) phase; thus, stars with masses similar to those considered here likely descend from 1-1.2 $\mathrm{M}_{\odot}$ progenitors.

The evolution of these objects is characterised by the gradual loss of the external mantle, which is accompanied by the growth in the core mass, which in this specific case, as shown in the left panel of Figure 1, rises from $\sim 0.53 \mathrm{M}_{\odot}$, at the beginning of the AGB phase, to $\sim 0.58 \mathrm{M}_{\odot}$, during the phases preceding the post-AGB evolution and the white dwarf cooling. Indeed, the evolution of these stars is driven by the growth of the core mass, which triggers a gradual increase in the luminosity of the star. This is in agreement with the classic study by [36], who demonstrated that a linear relation between the core mass and luminosity of AGB stars holds, as far as there is a radiative buffer separating the external envelope and the hydrogen-burning shell. This behaviour can be seen in the right panel of Figure 1, where we see the rise of the luminosity, from $\sim 2000 \mathrm{~L} \odot$ to $\sim 5500 \mathrm{~L} \odot$. 

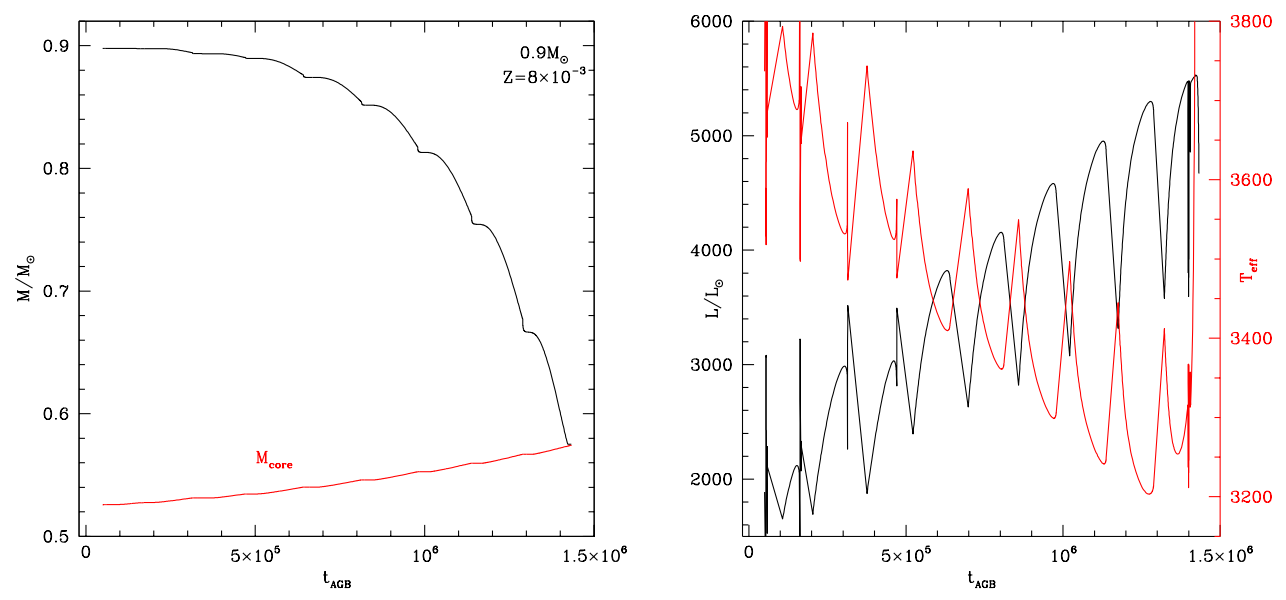

Figure 1. (Left) Variation during the AGB phase of the total mass (black line) and core mass (red) of a $0.9 \mathrm{M}_{\odot}$ star of metallicity $Z=8 \times 10^{-3}$. (Right) variation of luminosity (black, scale on the left) and effective temperature (red, scale on the right) for the same model star in the left panel.

The decrease in the mass of the envelope favours a general cooling of the external regions, with the effective temperatures diminishing by $\sim 500 \mathrm{~K}$ during the AGB lifetime. The simultaneous increase in the luminosities and cooling of the outermost regions make the star evolve on a more expanded configuration, with lower surface gravities, thus enhanced mass loss rates. The loss of the envelope becomes faster and faster, as can be seen in the left panel of Figure 1, until after seven TPs, the star begins the post-AGB evolution (this can be seen in the sudden increase in the effective temperature on the right side of the right panel of the figure).

No significant change in the surface chemical composition of low-mass stars is expected to take place during the AGB lifetime. The final surface chemistry reflects mainly the effects of the mixing processes occurring during the RGB ascending, discussed in Section 3, which favour the decrease in the abundance of ${ }^{12} \mathrm{C}$ and lithium and the rise in the surface mass fractions of helium and nitrogen and in the ${ }^{13} \mathrm{C} /{ }^{12} \mathrm{C}$ ratio $[37,38]$.

\subsection{Low-Mass C-Rich AGB Stars}

The stars with initial mass in the $1-3 \mathrm{M}_{\odot}$ range experience a higher number of TPs with respect to their lower-mass counterparts discussed earlier in this section, because their envelope is initially more massive; thus, a longer time is required before it is lost in its entirety. The most significant difference with respect to lower-mass stars is that the number of TPs experienced is sufficiently large such that the base of the convective envelope eventually penetrates inwards until reaching regions of the star previously contaminated by helium-burning nucleosynthesis [39]. This physical process, known as the third dredge-up (TDU), leads to a gradual enrichment in the surface carbon content and, to a lesser extent, in the oxygen abundance.

An example of the evolution of a model star belonging to this group is shown in Figure 2, which reports the time AGB variation of mass (both total and core mass), luminosity, and the surface mass fraction of the $\mathrm{CNO}$ elements as a function of the time elapsed since the start of the AGB phase. 

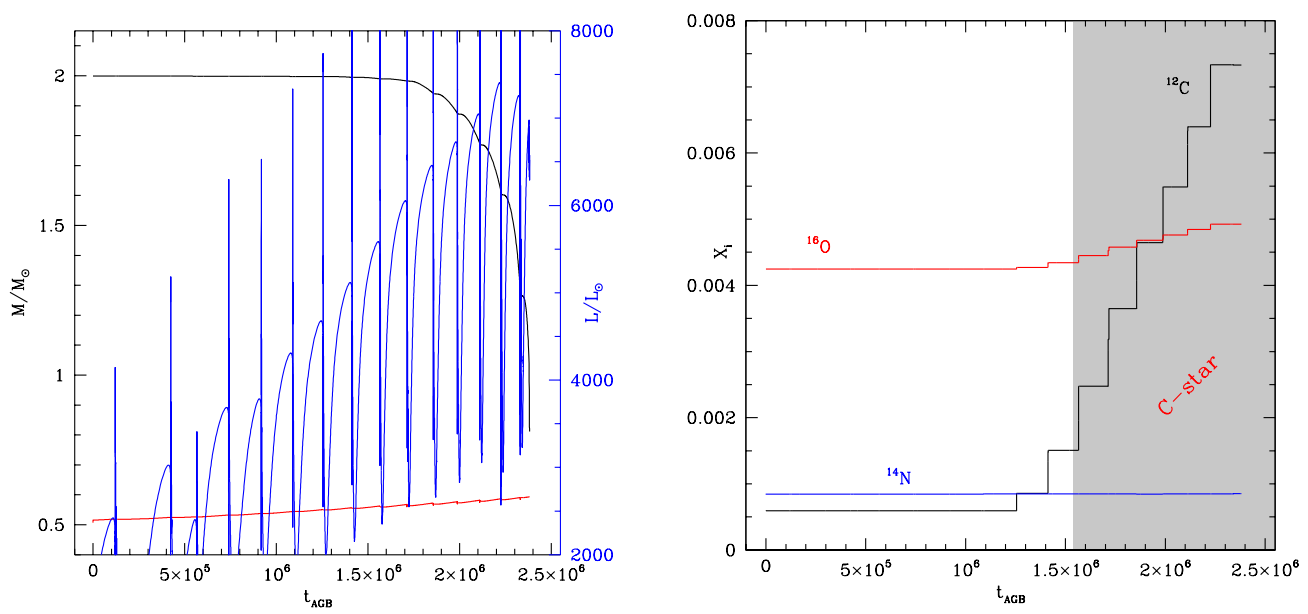

Figure 2. (Left) AGB evolution of the total and core mass (black and red lines, scale on the left) and of the luminosity (blue, scale on the right) of a $2 \mathrm{M}_{\odot}$ model star of metallicity $\mathrm{Z}=8 \times 10^{-3}$. (Right) variation of the surface $\mathrm{CNO}$ mass fractions; the grey-shaded area indicates the $\mathrm{C}$-star phase.

These stars, similar to their lower mass counterparts, obey the core mass-luminosity relationship by [36] discussed previously; thus, the luminosity increases as the core mass grows bigger. In the specific case reported in Figure 2, the luminosity reaches $\sim 7500 \mathrm{~L} \odot$ during the final AGB stages. The effects of the TDU can be recognised in the right panel of Figure 2, in the steep rise in the carbon mass fraction following each TP. When the number density of carbon particles exceeds that of oxygen, the star enters the carbon star stage. In the case shown in Figure 2, this takes place during the final part of the AGB phase (outlined with grey shadowing), whereas for the initial $\sim 70 \%$ of the AGB lifetime, the star evolves as an M-type star.

The achievement of the C-star stage has important consequences on the evolution of AGB stars. Once the $\mathrm{C} / \mathrm{O}>1$ condition is reached, the surface molecular opacities increase significantly [40], favouring the expansion and cooling of the surface regions [41,42] and the consequent formation of large amounts of dust [43]. All these factors lead to a fast increase in the mass loss rate, which causes the rapid loss of the external mantle. This effect can be seen in the left panel of Figure 2, where we note that the rate with which the mass of the star diminishes increases during the final AGB phases.

On the chemical side, the increase in the mass loss rate during the C-star phase has two important consequences: (a) the fast loss of the envelope prevents the star from experiencing a very large number of TPs and TDU events, thus the surface carbon mass fraction can hardly exceed $\sim 1 \%$ in all cases; (b) because most of the mass is lost after the C-star stage is reached, the chemical composition of the gas expelled from these stars is on average significantly enriched in carbon.

\subsection{Massive AGB Stars}

When the core mass of AGB stars exceeds $\sim 0.8 \mathrm{M}_{\odot}$ [44], the density and temperature gradients become so steep that the innermost regions of the convective envelope are partially overlapped with the CNO-burning shell, which favours the ignition of proton capture nucleosynthesis at the bottom of the envelope, a phenomenon known as hot bottom burning (HBB) [45]. The limit in the core mass given above corresponds to a minimum initial mass of $\sim 3 \mathrm{M}_{\odot}$, the exact value being sensitive to the assumptions regarding core overshoot during the main sequence evolution. This limit is also partly dependent on the metallicity of the star, as will be discussed in the following sections.

The activation of HBB leads to a significant increase in the overall luminosity of the star, far in excess (up to $\sim 4$-times higher in the most extreme cases) of the predictions based on the classic core mass-luminosity relationship by [36], discussed in Section 4.1 [46]. Before entering the discussion on the main properties of the stars experiencing HBB, we believe it 
important to stress once more that the results regarding the ignition and the strength of the HBB experienced by massive AGB stars are critically dependent on the description of the convective instability, particularly on the modality with which the temperature gradient is calculated in regions unstable to convective motions $[8,47]$.

Figure 3 shows the evolution of a $5 \mathrm{M}_{\odot}$ model star with the same metallicity, $Z=8 \times 10^{-3}$, as the models presented in Figures 1 and 2 . In the top-left panel of the figure, we can clearly see the deviation from the core mass-luminosity relationship discussed previously and the significant increase in the luminosity of the star. In the specific case shown in the figure, the largest luminosities are of the order of 50,000 $\mathrm{L}_{\odot}$; in highermass and/or lower-metallicity stars, the largest luminosities reached exceed $10^{5} \mathrm{~L}_{\odot}$ [19]. In the same panel of Figure 3, we recognise the typical evolution of the luminosity of massive AGB stars, which increases during the initial part of the AGB phase, until reaching a maximum value, then declines, as HBB is gradually turned-off by the loss of the external mantle.
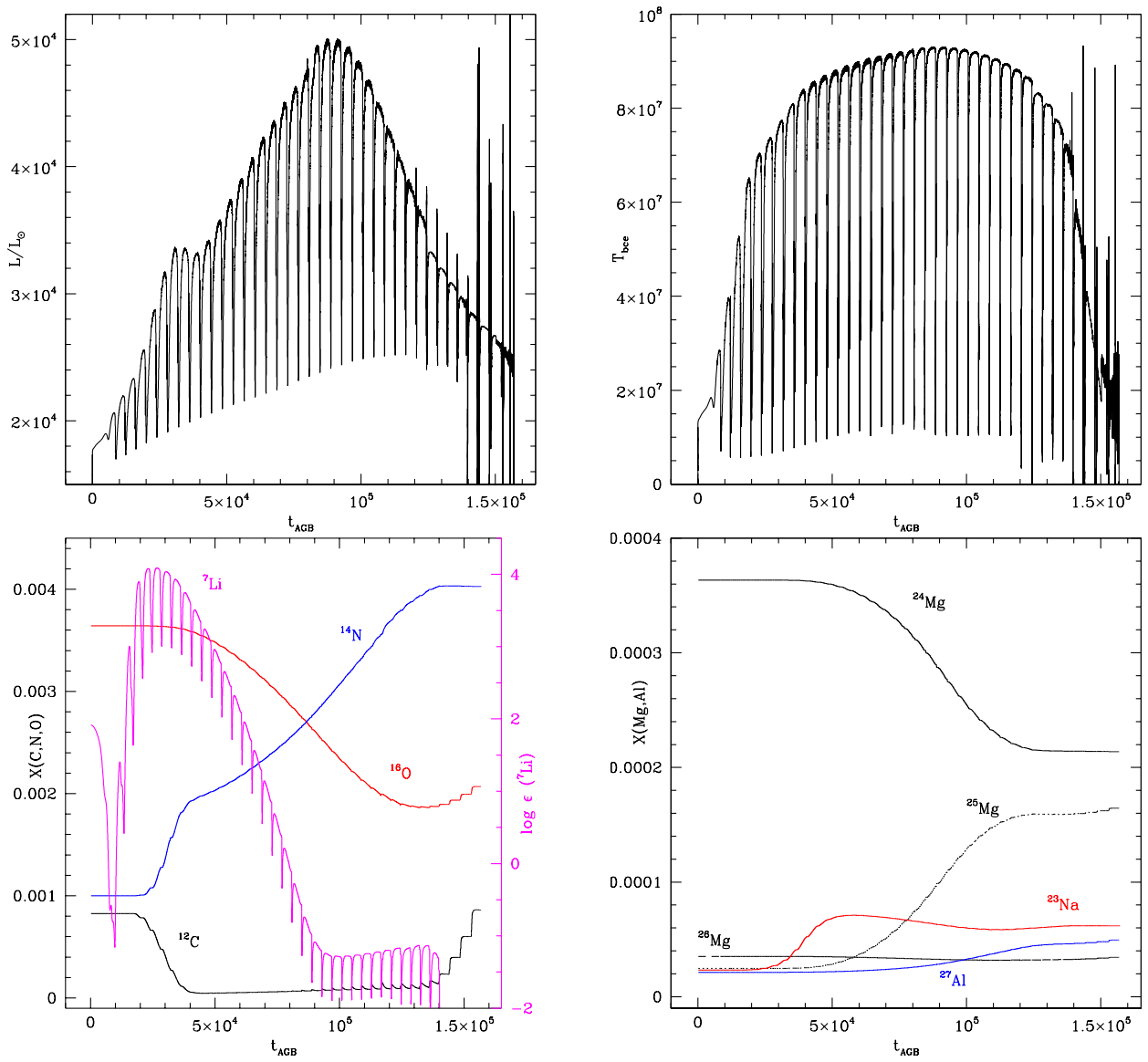

Figure 3. Time variation of luminosity (top, left panel), temperature at the base of the envelope (top, right), CNO mass fractions and the surface lithium in the $\log \epsilon\left({ }^{7} \mathrm{Li}\right.$ ) scale (where $\log \epsilon\left({ }^{7} \mathrm{Li}\right)=12+\log \left[\mathrm{n}\left({ }^{7} \mathrm{Li}\right) / \mathrm{n}(\mathrm{H})\right]$, scale on the right) (bottom, left), and Na-Mg-Al abundances (bottom, right) of a $5 \mathrm{M}_{\odot}$ model star of metallicity $\mathrm{Z}=8 \times 10^{-3}$.

On the chemical side, the activation of HBB triggers a fast decline in the surface carbon and the parallel increase in the nitrogen content. This can be seen in the bottom, left panel of Figure 3, where the anti-correlation between the ${ }^{12} \mathrm{C}$ and ${ }^{14} \mathrm{~N}$ behaviours is particularly clear in the $\sim 2-4 \times 10^{4}$ y time interval ${ }^{2}$. These phases are also characterised by the synthesis of large quantities of lithium, which is produced by means of the CameronFowler mechanism $[48,49]$. The super-rich lithium phase, which can be broadly defined as the time interval during which $\log \epsilon\left({ }^{7} \mathrm{Li}\right)>2$, lasts until there is some residual ${ }^{3} \mathrm{He}$ in the envelope. During the second part of the AGB phase, when the temperature at the bottom of the convective envelope exceeds $\sim 80 \mathrm{MK},{ }^{16} \mathrm{O}$ burning via the ${ }^{16} \mathrm{O}(\mathrm{p}, \gamma){ }^{17} \mathrm{~F}$ reaction 
takes place, which eventually favours a further increase in the ${ }^{14} \mathrm{~N}$ content. No further depletion of ${ }^{12} \mathrm{C}$ takes place after the beginning of ${ }^{16} \mathrm{O}$ burning, as the carbon equilibrium abundance increases when the full CNO cycling is activated. In the very late phases, we note the rise in the surface mass fraction of ${ }^{12} \mathrm{C}$ and ${ }^{16} \mathrm{O}$ : this is due to the effects of a few TDU events that take place after HBB is turned off, as a consequence of the gradual loss of the convective mantle.

In the bottom, right panel of Figure 3, we show the surface abundances of other species involved in proton capture nucleosynthesis, namely magnesium, sodium, and aluminium. We see the depletion of the surface ${ }^{24} \mathrm{Mg}$, which starts shortly after the ignition of ${ }^{16} \mathrm{O}$ burning, and the parallel increase in the ${ }^{25} \mathrm{Mg}$ and ${ }^{27} \mathrm{Al}$ content. The surface sodium also increases, a signature of the activation of the $\mathrm{Ne}-\mathrm{Na}$ chain.

While the activation of the $\mathrm{CN}$ nucleosynthesis and the synthesis of lithium is a general feature of the evolution of the stars experiencing HBB, the occurrence of a more advanced nucleosynthesis affecting the surface mass fractions of the other species from ${ }^{16} \mathrm{O}$ to ${ }^{28} \mathrm{Si}$ is extremely sensitive to the metallicity of the star [50]. Further discussion on the metallicity effects will be addressed in the following sections.

\subsection{Pollution from AGB Stars: General Trend with the Stellar Mass}

The results presented earlier in this section outline that the main properties of AGB stars are tightly connected with the initial mass. On the physical side, this regards primarily the luminosity reached by the stars and therefore the AGB lifetime. We saw earlier in this section that the luminosity range attained during the AGB lifetime is $3000-50,000 \mathrm{~L} \odot$, which corresponds to typical evolutionary AGB time-scales from a few $10^{4} \mathrm{y}$, for the brightest and highest-mass stars, to a few $10^{6} \mathrm{y}$, for the lowest-luminosity AGBs. These numbers refer to the $Z=8 \times 10^{-3}$ metallicity considered in the examples presented in Figure 1-3. In the metal-poor domain, massive AGBs evolve at higher luminosities, which reach $\sim 2 \times 10^{5} \mathrm{~L} \odot$ in the ultra-metal-poor cases [19].

As concerns the variation of the surface chemical composition, the role of the initial mass is extremely important, as we showed that it is the key parameter determining the relative importance of the TDU and HBB in modifying the surface chemistry during the AGB phase: low-mass stars experience the TDU only, whereas the higher-mass counterparts are exposed to HBB. This in turn affects the pollution from low- and intermediate-mass stars, because it is during the AGB phase that most of the mass of the envelope is removed from the star.

To discuss the chemical composition of the ejecta from AGB stars of various masses, we show in Figure 4 the gas yields of various chemical species of $Z=8 \times 10^{-3}$ stars. We follow the classical definition of the stellar yield, i.e., the yield of a given element $i$ is the net amount of the newly produced element that is ejected in the interstellar medium by a star during its life:

$$
\mathrm{Y}_{i}=\int\left[\mathrm{X}_{i}-\mathrm{X}_{i}^{\mathrm{init}}\right] \dot{M} d t
$$

The integral is calculated over the entire stellar lifetime; $X_{i}^{\text {init }}$ is the mass fraction of species $i$ at the beginning of the evolution; $\dot{M}$ is the mass loss rate.

The ${ }^{12} \mathrm{C}$ yields are the most sensitive to the stellar mass. In the $\mathrm{M} \leq 3 \mathrm{M} \odot$ domain, $\mathrm{Y}\left({ }^{12} \mathrm{C}\right)$ increases with the mass of the star, as the higher the initial mass, the larger the number of TDU events experienced, and the higher the surface carbon enrichment: $2.5-3 \mathrm{M}_{\odot}$ are those experiencing the largest carbon enrichment, with carbon yields slightly below $0.02 \mathrm{M}_{\odot}$. A clear discontinuity in $\mathrm{Y}\left({ }^{12} \mathrm{C}\right)$ is seen at $3.5 \mathrm{M}_{\odot}$, owing to the ignition of $\mathrm{HBB}$ in $\mathrm{M} \geq 3.5 \mathrm{M}_{\odot}$ stars. HBB favours the destruction of the surface ${ }^{12} \mathrm{C}$ (see the bottom, left panel of Figure 3); thus, the carbon yields turn negative, down to $\sim-0.006 \mathrm{M}_{\odot}$ for the largest masses considered, which are exposed to the strongest HBB conditions. 

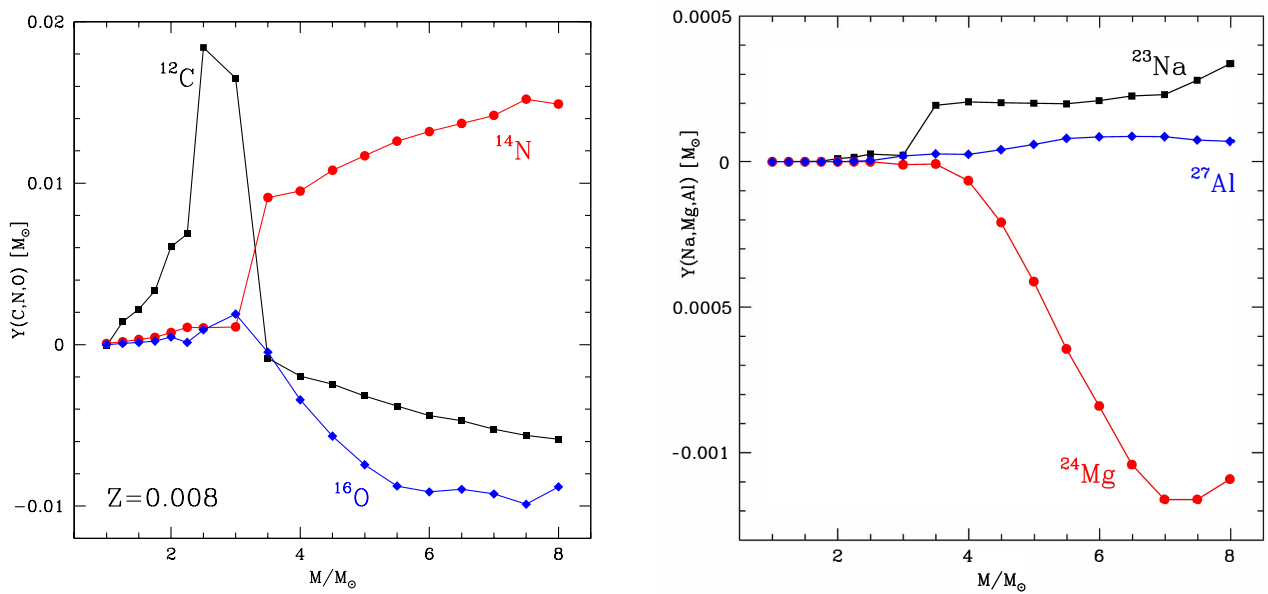

Figure 4. Yields of the most abundant isotopes involved in the CNO nucleosynthesis (left panel) and of sodium, magnesium, and aluminium (right) of $Z=8 \times 10^{-3}$ stars of different initial masses.

The oxygen yields on qualitative grounds follow the same behaviour of carbon. In the low-mass domain, the sensitivity to the initial mass of the star is smaller than carbon, because the amount of ${ }^{16} \mathrm{O}$ transported to the surface via the TDU is significantly smaller than ${ }^{12} \mathrm{C}$ (see the right panel of Figure 2). In the case of massive AGBs, the (negative) trend of $Y\left({ }^{16} \mathrm{O}\right)$ is steeper than $Y\left({ }^{12} \mathrm{C}\right)$ : this is because the rate of the ${ }^{16} \mathrm{O}$ destruction is sensitive to the temperature at the base of the envelope, hence to the mass of the star, whereas as discussed in Section 5.2, the equilibrium abundance of ${ }^{12} \mathrm{C}$ is not sensitive to the temperature, under $\mathrm{HBB}$ conditions involving the whole $\mathrm{CNO}$ cycling (see the bottom, left panel of Figure 3).

The nitrogen yields also exhibit a clear discontinuity around the threshold mass required to activate $\mathrm{HBB}$ : for $\mathrm{M}<3.5 \mathrm{M}_{\odot}$, we find a small production of ${ }^{14} \mathrm{~N}$, due to the effects of the FDU experienced during the RGB phase, as discussed in Section 3. Significant nitrogen production takes place when $\mathrm{HBB}$ is activated: the corresponding ${ }^{14} \mathrm{~N}$ yields span the $0.01-0.015 \mathrm{M}_{\odot}$ range, when the initial mass increases from $3.5 \mathrm{M}_{\odot}$ to $8 \mathrm{M}_{\odot}$.

The chemical species heavier than oxygen are mostly affected by HBB at these metallicities; thus, neither production nor destruction are expected in the low-mass domain. A deviation from this general behaviour, which will be discussed in the following section, is found in ultra-metal-poor stars. Indeed, in the right panel of Figure 4, we see that alterations in the gas composition as concerns $\mathrm{Na}, \mathrm{Mg}$, and $\mathrm{Al}$ is limited to masses above $3 \mathrm{M}_{\odot}$. The ejecta of these stars are enriched in sodium, which is produced by ${ }^{22} \mathrm{Ne}(\mathrm{p}, \gamma)^{23} \mathrm{Na}$ reactions at the base of the convective envelope and in ${ }^{27} \mathrm{Al}$, consequently to proton captures on magnesium nuclei; the activation of the $\mathrm{Mg}-\mathrm{Al}$ nucleosynthesis is further confirmed by the negative yields of ${ }^{24} \mathrm{Mg}$, which decrease down to $\sim-0.001 \mathrm{M}_{\odot}$ for $8 \mathrm{M}_{\odot}$ stars.

The results discussed in the present section are intended to give a broad indication of how the yields of the different chemical species change with the mass of the star. However, the yields are extremely sensitive to the metallicity of the star. This regards in particular the threshold mass required to activate $\mathrm{HBB}$, which increases with $Z$, and the effects of HBB itself, which are more pronounced at the lower metallicities; this concerns, e.g., the negative yields of oxygen and magnesium and the positive yields of sodium.

\section{Chemical Pollution from AGB Stars of Different Metallicities}

The results presented so far give a general idea of how AGB stars evolve and the modality with which they contaminate their surroundings, with gas altered with respect to the original chemical composition by different physical mechanisms. However, a thorough understanding of the gas pollution from this class of stars demands considering the role of metallicity, which affects the evolution across the AGB in different ways, related to the initial chemical composition of the different species, the internal temperature and density 
stratifications, the surface opacities, the equilibrium radius of the stellar structures, and the mass loss rates experienced.

\subsection{How Metallicity Affects the Evolution of Low-Mass AGBs}

We showed in Section 4.2 that in the low-mass domain, the TDU is the only mechanism able to alter the surface chemical composition of the stars, which becomes progressively more and more enriched in carbon. While this can be considered as the general behaviour of low-mass stars, the amount of carbon that is accumulated in the surface regions and the time fraction during which the stars evolve as carbon stars are sensitive to the metallicity.

In Figure 5, we consider model stars of different metallicity, with an initial mass typical of stars undergoing significant carbon enrichment at the surface, i.e., $2 \mathrm{M}_{\odot}$. The panels show the evolution of the surface ${ }^{12} \mathrm{C}$ mass fraction and of the $\mathrm{C} / \mathrm{O}$ ratio (the latter is in log units, to allow showing the results corresponding to the different $Z$ 's on the same plane). The variation of the surface carbon is shown as a function of the current mass of the star, to have an idea of the carbon content of the gas ejected into the surroundings of the star. On the other hand, to have an idea of the fraction of time spent in the C-star phase, the variation of the $\mathrm{C} / \mathrm{O}$ ratio is reported as a function of the AGB lifetime, normalised to the total duration of the AGB phase. For the sake of clarity, we limited this analysis to four metallicities, extending from the extremely metal-poor domain $\left(Z=3 \times 10^{-5}\right)$ to super-solar metallicities $(Z=0.03)$.
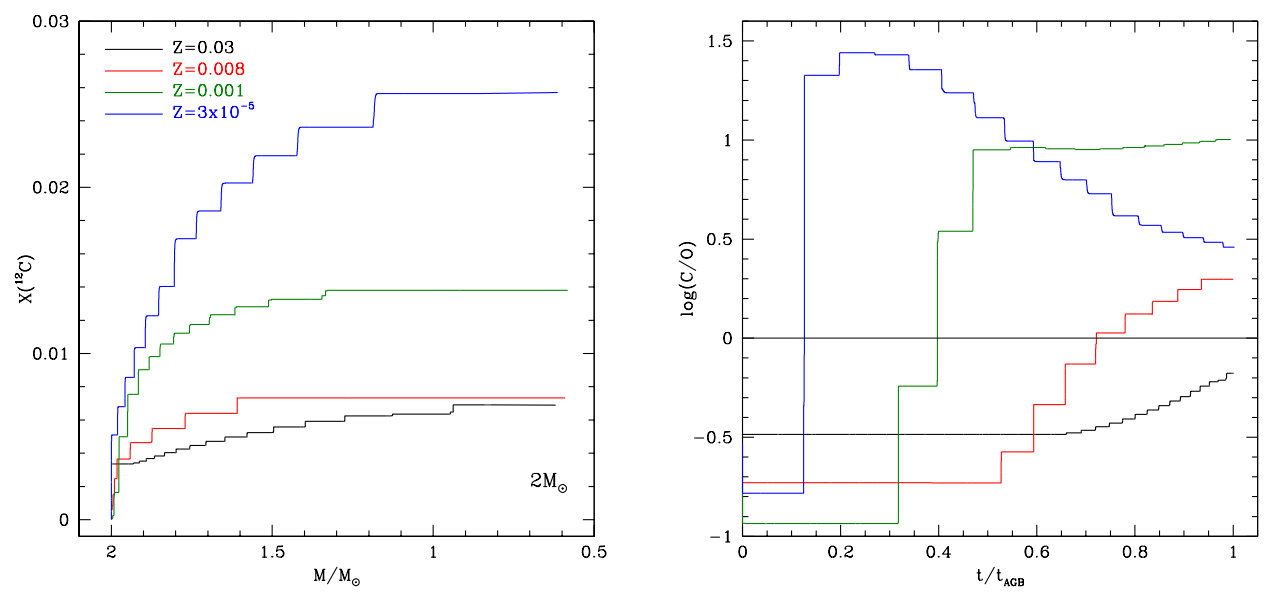

Figure 5. Variation of the surface mass fraction of ${ }^{12} \mathrm{C}$ (left panel) and of the $\mathrm{C} / \mathrm{O}$ ratio (right, logarithmic scale) of model stars of initial mass $2 \mathrm{M}_{\odot}$ and different metallicities, extending from the ultra-metal-poor domain to super-solar chemistries. The former quantity is shown as a function of the current mass of the star, whereas the carbon-to-oxygen ratio is reported as a function of the AGB lifetime, normalised to the overall duration of the AGB phase. The thin horizontal line in the right panel indicates the $\mathrm{C}=\mathrm{O}$ condition at the surface, thus delimiting $\mathrm{M}$-stars and carbon stars.

It is clear from the results shown in the right panel of Figure 5 that the lower the metallicity of the star, the earlier the C-star stage is reached and the higher the fraction of AGB time during which the star evolves as a C-star. The reason for this trend is the lower initial oxygen content in low- $Z$ stars, which eases the achievement of the $\mathrm{C} / \mathrm{O}>1$ condition. We note the significant differences among the results corresponding to different $Z$ 's: in the $Z=3 \times 10^{-5}$ case, the star evolves as a C-star for almost the entire AGB lifetime, while the $Z=0.03$ model star never becomes a C-star.

The metallicity of the stars also affects the amount of carbon that can be accumulated to the surface, as shown in the left panel of Figure 5, where we see that the final surface carbon abundance, decreasing with the metallicity, ranges from $\sim 0.007$, for $Z=0.03$, to $\sim 0.025$, for $Z=3 \times 10^{-5}$. The reasons for this behaviour are related to the reaction of the surface regions of the star to the enrichment in carbon and to the differences between the mass loss rates suffered by stars of similar mass and different metallicities. We discussed 
in Section 4.2 that the transition from an M-type to C-star is accompanied by a significant increase in the surface molecular opacities, which triggers the expansion of the surface regions and the consequent enhancement in the mass loss rate experienced. This partly inhibits the accumulation of carbon in the surface regions, as the envelope of the star is quickly removed, thus limiting the number of further TDU events experienced. The structure of low-metallicity stars is more compact; thus, the stellar radii and the mass loss rates corresponding to a given surface carbon mass fraction are smaller than in the higher-metallicity counterparts: therefore, the number of TDU events experienced by low-metallicity stars is higher, which is the reason for the higher final surface carbon abundances attained.

\subsection{The Connection between the Strength of the HBB of Massive AGBs and Metallicity}

Figure 6 shows the largest luminosity and temperature at the base of the envelope attained by stars of different masses and metallicities, chosen in the $Z=3 \times 10^{-7}-0.03$ domain. The results shown in Figure 6 confirm previous findings that lower-metallicity stars are generally brighter than the higher- $Z$ counterparts and are characterised by hotter temperatures at the bottom of the external envelope [44].
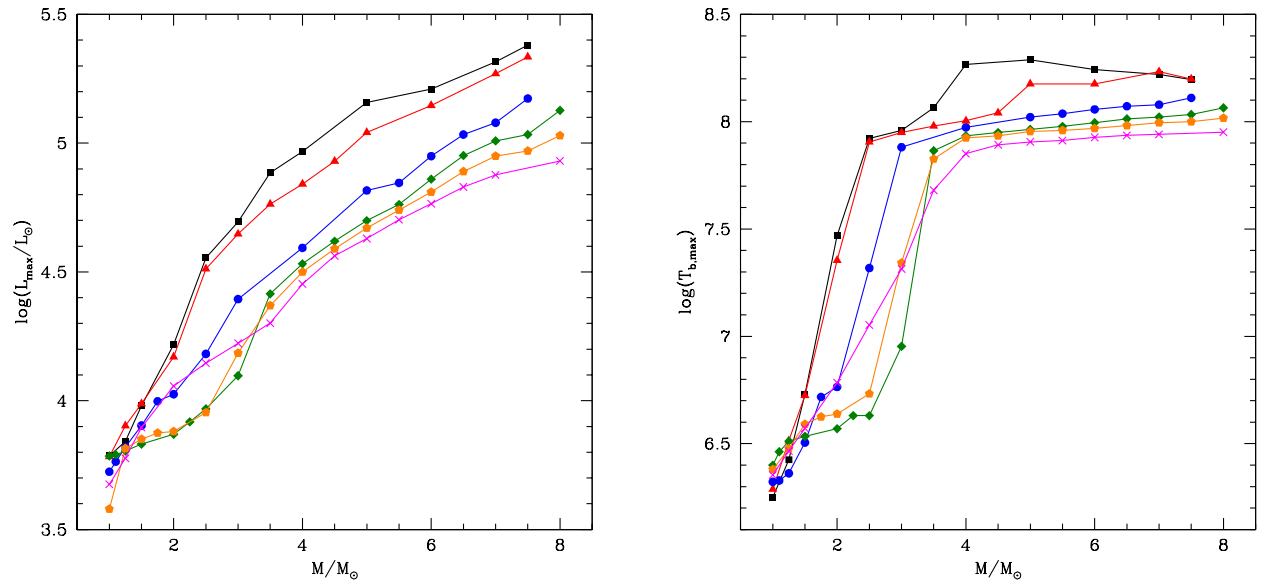

Figure 6. The largest luminosities (left panel) and temperatures at the bottom of the envelope (right) attained by AGB stars, as a function of the initial mass. The various colours and symbols refer to the metallicities $Z=3 \times 10^{-7}$ (black squares), $Z=3 \times 10^{-5}$ (red triangles), $Z=10^{-3}$ (blue points), $Z=8 \times 10^{-3}$ (green diamonds), solar metallicity $(Z=0.014$, orange pentagons), and $Z=0.03$ (magenta crosses).

The metallicity affects the minimum threshold mass required to activate HBB: in the ultra-metal-poor domain, $\mathrm{HBB}$ conditions are reached in $\mathrm{M}>2 \mathrm{M}_{\odot}$ stars [19], whereas in the super-solar case, the ignition of $\mathrm{HBB}$ requires a minimum mass of $\sim 3.5 \mathrm{M}_{\odot}$ [20].

The strength of the HBB experienced is also sensitive to the metallicity of the star. The nucleosynthesis that takes place at the bottom of the envelope of metal-poor, massive AGB stars is more advanced than in the metal-rich domain. As shown in the right panel of Figure 6, the temperatures reached at the base of the envelope barely reach $100 \mathrm{MK}$ in super-solar-metallicity AGBs, whereas they are $200 \mathrm{MK}$ in the ultra-metal-poor domain.

These differences affect the evolution of the surface chemistry of the stars, therefore the chemical composition of the ejecta. To discuss this point, we show in Figure 7 the variation of the surface mass fraction of ${ }^{16} \mathrm{O},{ }^{23} \mathrm{Na}$, and ${ }^{27} \mathrm{Al}$ during the AGB phase of $5 \mathrm{M}_{\odot}$ model stars of the same metallicities discussed in Figure 5. The quantity shown is the ratio of the mass fraction of the individual species considered with respect to the initial quantity, to have an idea of the percentage variation. We did not consider carbon and nitrogen here, because $\mathrm{CN}$ cycling is operative under HBB conditions, independently of metallicity; conversely, the occurrence and the efficiency of proton capture reactions involving heavier species is highly sensitive to $Z$ [50]. 
The evolution of the surface oxygen is tightly connected with the temperature at which the nucleosynthesis takes place, as ${ }^{16} \mathrm{O}$ is exposed to proton captures at the base of the envelope, while there is no reaction producing ${ }^{16} \mathrm{O}$ efficiently: therefore, oxygen is destroyed at the surface of massive AGBs across the whole AGB phase. The significant sensitivity of the ${ }^{16} \mathrm{O}$ depletion with metallicity is clear: the surface oxygen is practically unchanged in the $Z=0.03$ case, whereas it is almost completely destroyed in the envelope of the $3 \times 10^{-5}$ model star.
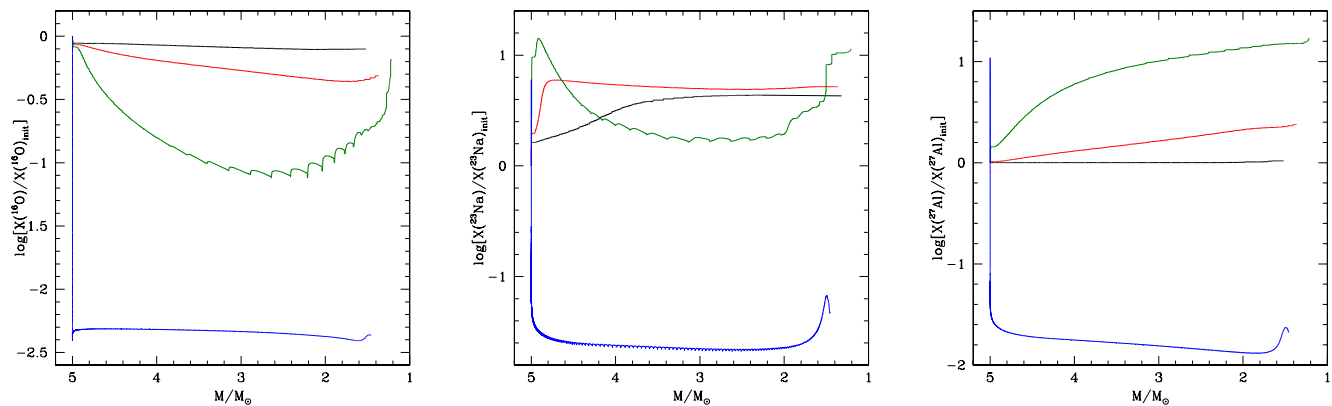

Figure 7. Variation of the surface mass fraction of ${ }^{16} \mathrm{O}$ (left panel), ${ }^{23} \mathrm{Na}$ (middle), and ${ }^{27} \mathrm{Al}$ (right) during the AGB phase of $5 \mathrm{M}_{\odot}$ model stars of the same metallicities presented in Figure 5: $Z=3 \times 10^{-5}$ (blue line), $Z=10^{-3}$ (green), $Z=8 \times 10^{-3}$ (red), and $Z=0.03$ (black). On the abscissa, we report the current mass of the star. The various mass fractions are reported with respect to the initial abundances.

The behaviour of sodium, which reflects the activation of the $\mathrm{Ne}-\mathrm{Na}$ chain, is more tricky, as the equilibrium surface mass fraction arises from the balance between the formation and destruction channels [51]. Significant sodium production takes place in the $Z=0.03$ and $Z=8 \times 10^{-3}$ cases, as the temperatures reached at the base of the envelope are sufficiently large to activate the ${ }^{22} \mathrm{Ne}(\mathrm{p}, \gamma)^{23} \mathrm{Na}$ reaction, at rates much higher than the proton captures ${ }^{23} \mathrm{Na}(\mathrm{p}, \gamma){ }^{24} \mathrm{Mg}$ and ${ }^{23} \mathrm{Na}(\mathrm{p}, \alpha){ }^{20} \mathrm{Ne}$. In the metal-poor domain, the latter destruction channels take over; thus sodium, after a short, initial production phase, is destroyed in the surface regions of the star.

Note that the surface abundances of both sodium and oxygen increase during the very final phases, owing to the effects of late TDU events, which become the only mechanism able to alter the surface chemical composition after HBB is turned off.

The surface mass fraction of ${ }^{27} \mathrm{Al}$ reflects the activation of the $\mathrm{Mg}-\mathrm{Al}$ chain. The poor aluminium production occurring at super-solar metallicities is favoured by proton captures by the heavier and less-abundant isotopes of magnesium, namely ${ }^{25} \mathrm{Mg}$ and ${ }^{26} \mathrm{Mg}$; the temperatures in this case are not sufficient to favour the destruction of the most abundant isotope ${ }^{24} \mathrm{Mg}$, which is required to reach large $\mathrm{Al}$ production. As the metallicity decreases, the ${ }^{27} \mathrm{Al}$ production increases, as the depletion of ${ }^{24} \mathrm{Mg}$ takes over. Note that the surface ${ }^{27} \mathrm{Al}$ barely grows beyond a factor of 10 , as the synthesis of silicon takes place in environments largely enriched in aluminium. In the ultra-metal poor domain, the HBB temperatures are so high that the nucleosynthesis is so advanced so as to favour the destruction of aluminium and silicon at the surface of the star.

\section{Gas Pollution from AGB Stars: A General Overview}

In the previous section, we examined the physical and chemical properties of AGB stars, particular focused on the modality with which the surface chemical composition changes in stars of different masses and metallicities. We now discuss the general pollution expected from these stars, focusing on the average chemical composition of the gas that they share with the interstellar medium across the whole AGB lifetime. We start with helium, then we focus on the CNO and other species involved in HBB nucleosynthesis. 


\subsection{Helium in the Ejecta of AGB Stars}

The gas expelled from AGB stars is enriched in helium. This is reported in Figure 8, which shows the average helium mass fraction of the ejecta of stars of various masses and metallicities. The small increase with respect to the initial abundance of the helium content of the ejecta of low-mass AGBs is due to the effects of the FDU (see the discussion in Section 3) and to the gradual surface helium enrichment, favoured by the $\mathrm{CNO}$ nucleosynthesis in the hydrogen-burning shell.

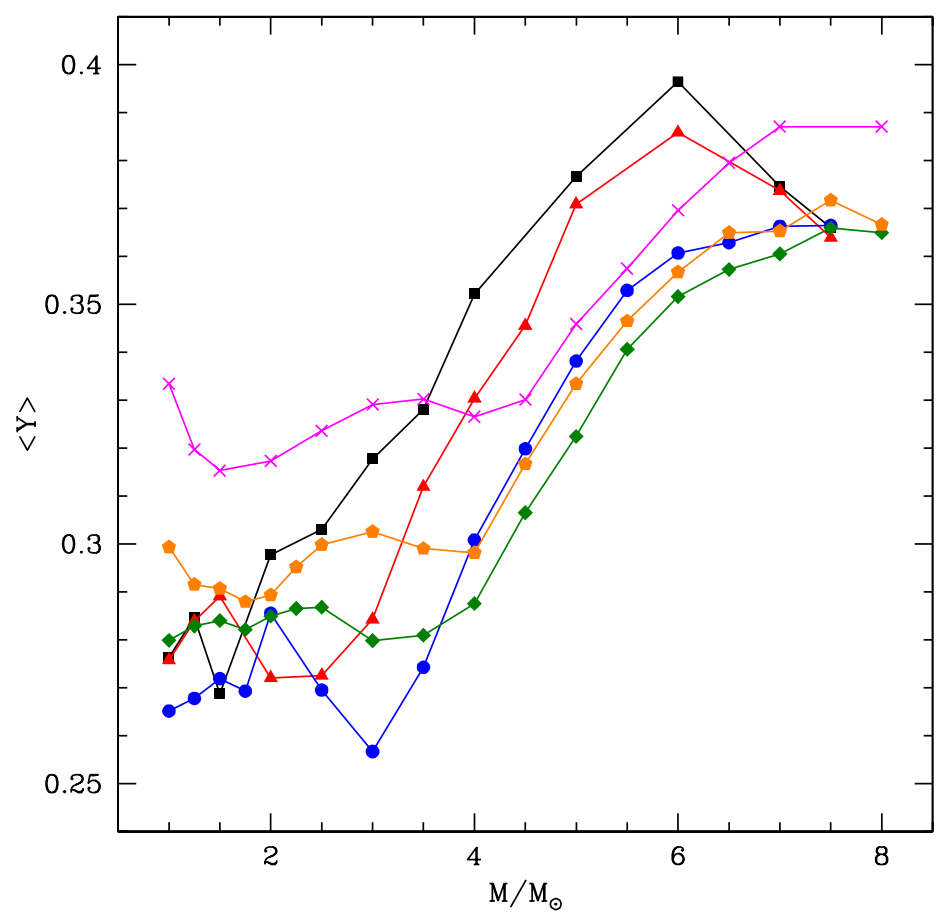

Figure 8. Average helium content of the ejecta of stars of various masses and metallicities. The average is obtained by the integral over the AGB time of the rate at which helium is lost by the star (in turn given by the product of the surface helium mass fraction and the mass loss rate of the star), normalised to the total mass lost by the star during the AGB phase. The colour coding adopted is the same as in Figure 6.

Stars with initial mass above $\sim 3 \mathrm{M}_{\odot}$ are exposed to a convective mixing episode taking place soon after the exhaustion of the central helium, known as the second dredge-up (SDU). As in the case of HBB, the minimum threshold mass required to experience the SDU is slightly sensitive to the metallicity and increases with $Z$. During the SDU, the external mantle of the star penetrates inwards, past the $\mathrm{H}-\mathrm{He}$ discontinuity; this process, which is made easier by the temporary extinction of the H-burning shell, favours the transportation of helium-rich material towards the surface layers, which become more and more enriched in helium [52].

The extent of the SDU increases with the mass of the star [53]: the helium enrichment associated with the second dredge-up reaches a maximum of $\delta \mathrm{Y} \sim 0.1$ for $7-8 \mathrm{M}_{\odot}$ stars. The ejecta of massive AGBs are characterised by a helium abundance close to $40 \%$ : this is the reason why these stars are considered as possible pollutants of the interstellar medium in Globular Clusters, providing the gas from which multiple stellar populations formed [53].

The predictions regarding the helium content of the ejecta are more robust than for the other chemical species, as most of the helium enrichment occurs during the SDU, taking place before the beginning of the TP phase, whose description is independent of all the uncertainties affecting AGB modelling. 


\subsection{The CNO Species}

To discuss the nucleosynthesis internal to AGB stars and the relative impact on the pollution of the interstellar medium, we show in Figure 9 the average mass fraction of different chemical species in the ejecta, with respect to the initial abundances. This choice allows understanding the extent of the production/destruction of a given element across the whole AGB phase, independent of the initial metallicity of the stars. We focus on the elements involved in the chemical reactions providing the largest contribution to the overall energy release and on other chemical species whose variation during the AGB phase is a key indicator of the efficiency of the chemical processes able to alter the surface chemical composition of these stars.
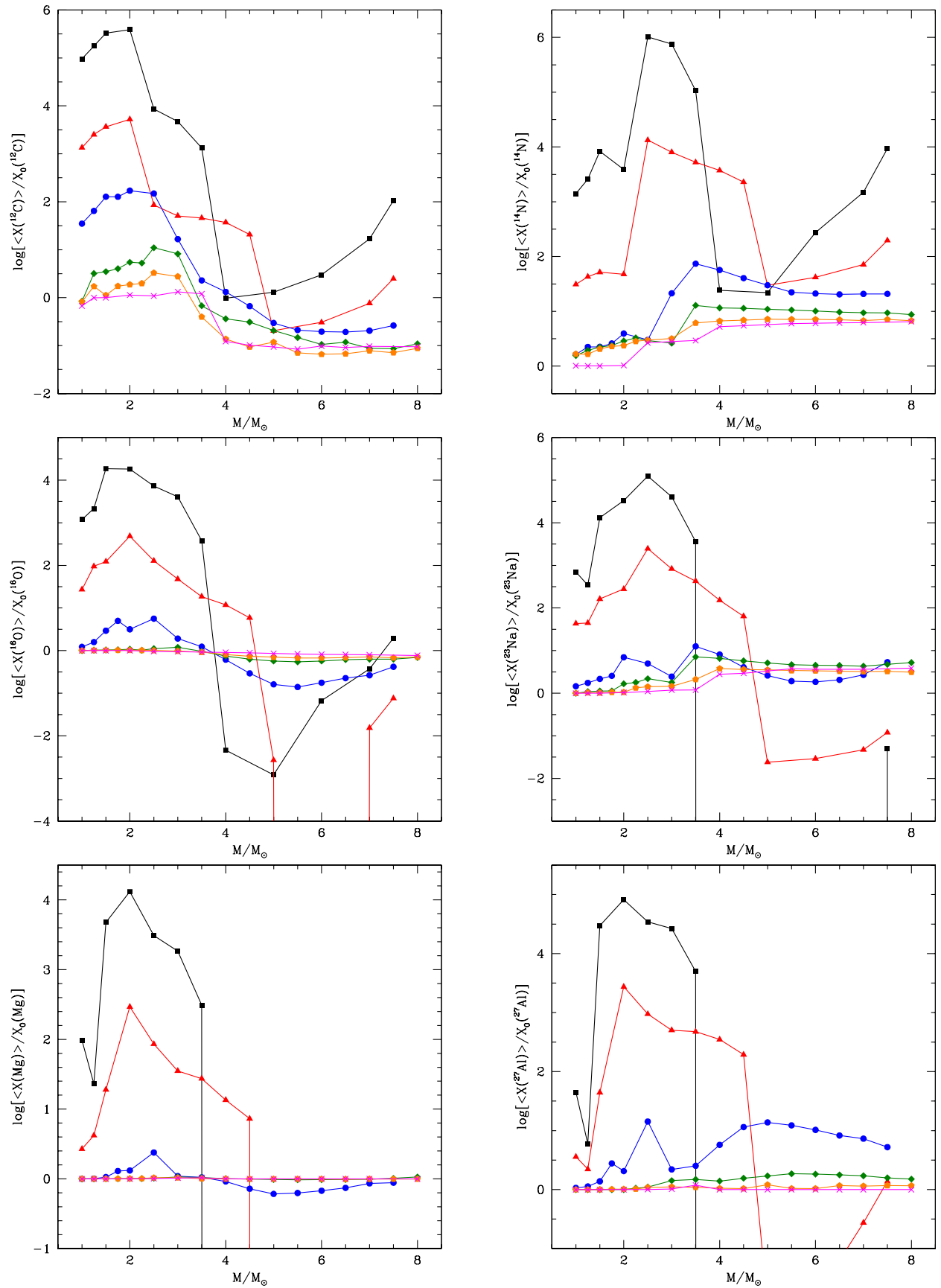

Figure 9. Ratio of the average abundance of different chemical species with respect to the initial mass fraction as a function of the initial mass of the star. The different colours and symbols have the same meaning as in Figures 6 and 8. 
The carbon yields shown in the top, left panel of Figure 9 exhibit clear trends with mass and metallicity, in agreement with the discussions in the previous sections. For each metallicity, we find that the average ${ }^{12} \mathrm{C}$ in the ejecta increases with the initial mass in the mass range of the stars not experiencing $\mathrm{HBB}$, which is limited to $\sim 2 \mathrm{M}_{\odot}$ in the ultra-metal-poor domain, whereas it is $\sim 3.5 \mathrm{M}_{\odot}$ in the super-solar-metallicity case. The production factor of carbon is higher in lower-metallicity stars: in the super-solar-metallicity domain, the carbon enrichment in the ejecta is below $20 \%$, whereas in the $Z=3 \times 10^{-5}$ and $Z=3 \times 10^{-7}$ cases, the average ${ }^{12} \mathrm{C}$ in the ejecta is four and six orders of magnitude higher than in the initial chemical composition, respectively. The reason for these differences is that the amount of carbon dredged up to the surface during each TDU event is substantially independent of metallicity, whereas the initial carbon abundance scales with Z. Low-mass, ultra-metal-poor AGBs are therefore efficient manufacturers of primary carbon.

In the mass domain of the stars experiencing $\mathrm{HBB}$, the yields are less sensitive to the metallicity, because the equilibrium carbon abundances under HBB conditions is proportional to the metallicity of the star. The mass of the star is not particularly relevant in this context, as the equilibrium carbon abundance is roughly independent of the HBB temperatures (see the bottom, left panel of Figure 3). The rising trend with mass in the carbon yields of 7-8 $\mathrm{M}_{\odot}$ stars is because after the end of core helium burning, the SDU is so deep that it reaches regions of the star enriched in carbon: this mechanism, known as dredge-out [54], favours the carbon enrichment of the surface regions.

On the qualitative side, the behaviour of the oxygen production factor is similar to carbon, but the sensitivity to the metallicity is higher. In the low-mass domain, a negligible ${ }^{16} \mathrm{O}$ enrichment is found for $Z \geq 8 \times 10^{-3}$, whereas in the metal-poor domain, the production factor increases towards the lowest metallicities considered, up to $\sim 10^{4}$ for $Z=3 \times 10^{-7}$. In the mass range of the stars experiencing $\mathrm{HBB}$, the oxygen in the ejecta is on average lower than in the gas from which the stars formed. This effect is negligible for $Z \geq 8 \times 10^{-3}$, because as shown in Section 5.2, the HBB experienced is not sufficiently vigorous to favour significant depletion of the surface ${ }^{16} \mathrm{O}$ in this metallicity domain. On the contrary, in metal-poor, massive AGB stars, the ignition of hot CNO cycling at the base of the envelope leads to oxygen-poor ejecta; in the ultra-metal-poor domain, the gas lost by the stars is practically oxygen-free. The rising trend of the ${ }^{16} \mathrm{O}$ production factor in the metal-poor, $\mathrm{M} \geq 5 \mathrm{M}_{\odot}$ stars is connected to the occurrence of the dredge-out, similar to what is found for ${ }^{12} \mathrm{C}$.

The nitrogen yields are also sensitive to mass and metallicity, but unlike carbon and oxygen, the associated production factor is above unity in all cases. In the ejecta of lowmass stars, from sub-solar to super-solar metallicity, some ${ }^{14} \mathrm{~N}$ enrichment is found, in agreement with the arguments discussed in Section 3, owing to the effects of the FDU. The corresponding production factor scales with metallicity and is of the order of $\sim 2-3$. In the higher-mass counterparts, the activation of $\mathrm{HBB}$ triggers the increase in the surface ${ }^{14} \mathrm{~N}$, which is enhanced by a factor $\sim 10$. In low-mass, metal-poor stars, the TDU is so efficient in modifying the surface chemistry that some ${ }^{14} \mathrm{~N}$ production via $\mathrm{p}$-captures by ${ }^{12} \mathrm{C}$ nuclei takes place, despite the relative cool temperatures at the base of the convective envelope. Of particular importance are the stars in the $2-4 \mathrm{M}_{\odot}$ mass domain, where significant amounts of primary nitrogen are produced at the expenses of the ${ }^{12} \mathrm{C}$ dredged up from the ashes of the helium-burning shell: these stars are among the most efficient manufactures of primary nitrogen. Even as concerns nitrogen, the rising trend of the ${ }^{14} \mathrm{~N}$ production factor with mass found in the most massive, low-metallicity AGBs is due to the effects of the dredge-out, which brings fresh ${ }^{12} \mathrm{C}$ to the surface, later converted into ${ }^{14} \mathrm{~N}$.

\subsection{From Sodium to Aluminium}

The sodium yields are sensitive to the amount of fresh ${ }^{22} \mathrm{Ne}$ transported to the surface by the TDU and to the ignition of the Ne-Na nucleosynthesis at the base of the envelope. As far as the effects of $\mathrm{HBB}$ are concerned, we see that the production factor of ${ }^{23} \mathrm{Na}$ is higher the higher the metallicity, because the HBB temperature increases at lower metallicities and 
the sodium equilibrium abundances diminish, as the destruction channels take over. This is consistent with the analysis presented in Section 5.2 and the results shown in Figure 7 (middle panel). In the most-metal-poor cases, the nucleosynthesis occurring at the base of the envelope is so efficient that the sodium content of the ejecta is extremely small. In the low-mass domain, sodium enrichment is found in the metal-poor case, owing to sodium synthesis by ${ }^{22} \mathrm{Ne}$ nuclei dredged up after each TP.

The magnesium and aluminium yields reflect the effects of mixing and of the HBB $\mathrm{Mg}-\mathrm{Al}$ nucleosynthesis. The magnesium content of the ejecta of $Z \geq 8 \times 10^{-3}$ stars is substantially unchanged with respect to the initial quantity, as the amount of $\mathrm{Mg}$ dredged up from the internal regions of the star is not sufficient to alter the surface abundance, and the HBB experienced, consistently with the results discussed in Section 5.2, is not sufficiently strong to activate proton captures by $\mathrm{Mg}$ nuclei. In the ${ }^{27} \mathrm{Al}$ case, some enrichment is expected in the ejecta in the solar and sub-solar cases, owing to the effects of proton captures by the heavier (and less abundant) magnesium isotopes, ${ }^{25} \mathrm{Mg}$ and ${ }^{26} \mathrm{Mg}$. In the ejecta of low-metallicity stars, both the $\mathrm{Mg}$ and ${ }^{27} \mathrm{Al}$ contents are significantly altered with respect to the initial quantities.

In low-mass stars, large amounts of magnesium are dredged up to the surface regions after each TP; thus, the ejecta are enriched in magnesium. The production factor is higher the lower the metallicity is, reaching $\sim 10^{4}$ in the ultra-metal-poor domain. The considerable increase in the surface $\mathrm{Mg}$ leads to the activation of the $\mathrm{Mg}-\mathrm{Al}$ chain even in the low-mass domain, with the production factor of ${ }^{27} \mathrm{Al}$ reaching $\sim 10^{4}$ for $Z=3 \times 10^{-7}$.

In metal-poor, massive AGBs, the activation of HBB leads to the depletion of the surface magnesium: the $\mathrm{Mg}$ content of the ejecta is a factor $\sim 2$ smaller than the initial abundance in $Z=10^{-3}$ stars, whereas in the ultra-metal-poor domain, the gas expelled by massive AGBs is practically magnesium-free. The trend of the ${ }^{27} \mathrm{Al}$ production factor of massive AGBs with metallicity is more tricky. When the metallicity decreases to $Z=10^{-3}$, the ${ }^{27} \mathrm{Al}$ production factor is $\sim 10$. In the ultra-metal-poor domain, little ${ }^{27} \mathrm{Al}$ is present in the ejecta, as the HBB nucleosynthesis is shifted to heavier chemical species.

\section{Conclusions}

We discussed the evolution of 1-8 $\mathrm{M}_{\odot}$ stars across the AGB phase, until the beginning of the general contraction that leads to the post-AGB and Planetary Nebula stages. To study the impact of the chemical composition of the stars on the main general evolutionary properties and on the pollution of the interstellar medium, we considered a wide range of metallicities, extending from the ultra-metal-poor domain, with $Z=3 \times 10^{-7}$, to supersolar chemical compositions, with $Z=0.04$.

The initial mass of the star was confirmed as the most relevant quantity in determining the main evolutionary properties and the modification of the surface chemical composition. For each metallicity, a threshold-limiting mass of $\sim 2-3 \mathrm{M}_{\odot}$ separates low-mass stars from massive AGBs. The former experience a series of thermal pulses, followed by the occurrence of third dredge-up episodes, which eventually lead to the formation of carbon stars. The evolution of these stars is driven by the growth in the core mass, which in turn favours the increase in the overall luminosity, which eventually reaches $5000-12,000 \mathrm{~L} \odot$, the higher masses evolving to higher luminosities. On the other hand, massive AGBs experience hot bottom burning, which favours the destruction of the surface carbon and is associated with a fast increase in the luminosity, which in the largest mass stars approaches and overcomes $10^{5} \mathrm{~L} \odot_{\odot}$.

The metallicity of the stars is important to determine the evolution and particularly the chemistry of the ejecta of AGB stars. The aforementioned threshold mass separating the low-mass domain from massive AGBs changes from $\sim 2 \mathrm{M}_{\odot}$, for ultra-metal-poor chemistries, to $\sim 3.5 \mathrm{M}_{\odot}$, for super-solar chemical compositions.

The modification of the surface chemical composition of AGB stars, and the relative chemistry of the ejecta, is extremely sensitive to the metallicity. This is particularly true for massive AGBs, where the signatures of HBB nucleosynthesis changes considerably 
with the temperature at the base of the convective envelope, which in turn is highly sensitive to $Z$. This regards in particular the elements involved in advanced proton capture nucleosynthesis, such as ${ }^{16} \mathrm{O}$ and magnesium, whose abundance is practically unchanged with respect to the initial mass fraction in the ejecta of solar and super-solar stars, whereas it approaches zero in the ultra-metal-poor metallicity domain.

Author Contributions: Conceptualisation, P.V.; investigation, M.C., M.D.C., E.M., M.T. and S.T.; supervision, F.D.; writing-original draft, P.V. All authors have read and agreed to the published version of the manuscript.

Funding: This research received no external funding.

Acknowledgments: P.V. benefited from the International Space Science Institute (ISSI, Bern, CH, and ISSI-BJ, Beijing, CN) thanks to the funding of the team "Chemical abundances in the ISM: the litmus test of stellar IMF variations in galaxies across cosmic time"; E.M. acknowledges support from the Angelo Della Riccia foundation.

Conflicts of Interest: The authors declare no conflict of interest.

\section{Notes}

1 The masses given here and in the following sections refer to the beginning of the AGB phase. The masses of the progenitors are generally higher and are sensitive to the mass lost during the red giant branch phase.

2 The beginning of HBB and of the relative chemical signatures start earlier during the AGB phase the higher the initial mass of the star is.

\section{References}

1. Ferrarotti, A.; Gail, H.P. Composition and quantities of dust produced by AGB-stars and returned to the interstellar medium. Astron. Astrophys. 2006, 447, 553-576. [CrossRef]

2. Romano, D.; Karakas, A.I.; Tosi, M.; Matteucci, F. Quantifying the uncertainties of chemical evolution studies. II. Stellar yields. Astron. Astrophys. 2010, 522, A32. [CrossRef]

3. Romano, D.; Matteucci, F.; Zhang, Z.-Y.; Ivison, R.J.; Ventura, P. The evolution of CNO isotopes: The impact of massive stellar rotators. Mon. Not. R. Astron. Soc. 2019, 490, 2838. [CrossRef]

4. Vincenzo, F.; Belfiore, F.; Maiolino, R.; Matteucci, F.; Ventura, P. Nitrogen and oxygen abundances in the Local Universe. Mon. Not. R. Astron. Soc. 2016, 458, 3466-3477. [CrossRef]

5. D'Ercole A., Vesperini E., D'Antona F., McMillan S. L.W., Recchi S. Formation and dynamical evolution of multiple stellar generations in globular clusters. Mon. Not. R. Astron. Soc. 2008, 391, 825-843 [CrossRef]

6. Schwarzschild, M.; Härm, R. Thermal instability in non-degenerate stars. Astrophys. J. 1965, 142, 855. [CrossRef]

7. Karakas, A.I.; Lattanzio, J.C. The Dawes Review 2: Nucleosynthesis and Stellar Yields of Low- and Intermediate-Mass Single Stars. Publ. Astron. Soc. Aust. 2014, 31, 1-70. [CrossRef]

8. Ventura, P.; D'Antona, F. Full computation of massive AGB evolution. I. The large impact of convection on nucleosynthesis. Astron. Astrophys. 2005, 431, 279-288. [CrossRef]

9. Ventura, P.; Zeppieri, A.; Mazzitelli, I.; D'Antona, F. Full spectrum of turbulence convective mixing: I. theoretical main sequences and turn-off for 0.6-15 Msun. Astron. Astrophys. 1998, 334, 953-968.

10. Cloutman, L.D.; Eoll, J.G. Comments on the diffusion model of turbulent mixing. Astrophys. J. 1976, 206, 584. [CrossRef]

11. Canuto, V.M.C.; Mazzitelli, I. Stellar Turbulent Convection: A New Model and Applications. Astrophys. J. 1991, 370, 295. [CrossRef]

12. Bloecker, T. Stellar evolution of low and intermediate-mass stars. I. Mass loss on the AGB and its consequences for stellar evolution. Astron. Astrophys. 1995, 297, 727.

13. Wachter, A.; Schröder, K.-P.; Winters, J.M.; Arndt, T.U.; Sedlmayr, E. An improved mass loss description for dust-driven superwinds and tip-AGB evolution models. Astron. Astrophys. 2002, 384, 452. [CrossRef]

14. Wachter, A.; Winters, J.M.; Schröder, K.-P.; Sedlmayr, E. Dust-driven winds and mass loss of C-rich AGB stars with subsolar metallicities. Astron. Astrophys. 2008, 486, 497. [CrossRef]

15. Marigo, P.; Aringer, B. Low-temperature gas opacity. ÆSOPUS: A versatile and quick computational tool. Astron. Astrophys. 2009, 508, 1539. [CrossRef]

16. Ventura, P.; Karakas, A.I.; Dell'Agli, F.; García-Hernández, D.A.; Guzman-Ramirez, L. Gas and dust from solar metallicity AGB stars. Mon. Not. R. Astron. Soc. 2018, 475, 2282. [CrossRef]

17. Ventura, P.; Karakas, A.I.; Dell'Agli, F.; Boyer, M.L.; García-Hernández, D.A.; Criscienzo, M.D.; Schneider, R. The Large Magellanic Cloud as a laboratory for hot bottom burning in massive asymptotic giant branch stars. Mon. Not. R. Astron. Soc. 2015, 450, 3181. [CrossRef] 
18. Ventura, P.; Karakas, A.I.; Dell'Agli, F.; García-Hernández, D.A.; Boyer, M.L.; Criscienzo, M.D. On the nature of the most obscured C-rich AGB stars in the Magellanic Clouds. Mon. Not. R. Astron. Soc. 2016, 457, 1456. [CrossRef]

19. Ventura, P.; Dell'Agli, F.; Romano, D.; Tosi, S.; Limongi, M.; Chieffi, A.; Castellani, M.; Tailo, M.; Lugaro, M.; Marini, E.; et al. Gas and dust from extremely metal-poor AGB stars. Astron. Astrophys. 2021, 655, A6. [CrossRef]

20. Ventura, P.; Dell'Agli, F.; Lugaro, M.; Romano, D.; Tailo, M.; Yagüe, A. Gas and dust from metal-rich AGB stars. Astron. Astrophys. 2020, 614, A103. [CrossRef]

21. Mikolaitis, Š.; Tautvaišienè, G.; Gratton, R.; Bragaglia, A.; Carretta, E. Chemical composition of clump stars in the open cluster NGC 6134. Mon. Not. R. Astron. Soc. 2010, 407, 1866. [CrossRef]

22. Smiljanic, R.; Gauderon, R.; North, P.; Barbuy, B.; Charbonnel, C.; Mowlavi, N. CNONa and ${ }^{12} \mathrm{C} /{ }^{13} \mathrm{C}$ in giant stars of 10 open clusters. Astron. Astrophys. 2009, 502, 267. [CrossRef]

23. Origlia, L.; Valenti, E.; Rich, R.M. High resolution infrared spectra of NGC 6440 and NGC 6441: Two massive bulge globular clusters. Mon. Not. R. Astron. Soc. 2008, 388, 1419. [CrossRef]

24. Pilachowski, C.A.; Sneden, C.; Kraft, R.P.; Langer, G.E. Proton capture chains in Globular Cluster Stars. I. evidence for deep mixing based on sodium and magnesium abundances in M13 giants. Astron. J. 1996, 112, 545. [CrossRef]

25. Valenti, E.; Origlia, L.; Rich, R.M. High-resolution near-infrared spectra of NGC 6624 and 6569. Mon. Not. R. Astron. Soc. 2011, 414, 1690. [CrossRef]

26. Angelou, G.C.; Church, R.P.; Stancliffe, R.J.; Lattanzio, J.C.; Smith, G.H. Thermohaline Mixing and its Role in the Evolution of Carbon and Nitrogen Abundances in Globular Cluster Red Giants: The Test Case of Messier 3. Astrophys. J. 2011, 728, 79. [CrossRef]

27. Angelou, G.C.; Stancliffe, R.J.; Church, R.P.; Lattanzio, J.C.; Smith, G.H. The Role of Thermohaline Mixing in Intermediate- and Low-metallicity Globular Clusters. Astrophys. J. 2012, 749, 128. [CrossRef]

28. Mestel, L. Rotation and stellar evolution. Mon. Not. R. Astron. Soc. 1953, 113, 716. [CrossRef]

29. Maeder, A.; Meynet, G. Evolution of massive stars with mass loss and rotation. New Astron. Rev. 2010, 54, 32. [CrossRef]

30. Tassoul, M.; Tassoul, J.M. Meridional circulation in rotating stars. XI. Single-cell pattern versus double-cell pattern. Astrophys. J. 1995, 440, 789. [CrossRef]

31. Palacios, A.; Charbonnel, C.; Talon, S.; Siess, L. Rotational mixing in low-mass stars. II. Self-consistent models of Pop II RGB stars. Astron. Astrophys. 2006, 453, 261. [CrossRef]

32. Eggleton, P.P.; Dearborn, D.S.P.; Lattanzio, J.C. Deep Mixing of ${ }^{3}$ He: Reconciling Big Bang and Stellar Nucleosynthesis. Science 2018, 314, 1580. [CrossRef]

33. Busso, M.; Gallino, R.; Wasserburg, G.J. Nucleosynthesis in Asymptotic Giant Branch Stars: Relevance for Galactic Enrichment and Solar System Formation. Annu. Rev. Astron. Astrophys. 1999, 37, 239-309. [CrossRef]

34. Herwig, F. Evolution of Asymptotic Giant Branch Stars. Annu. Rev. Astron. Astrophys. 2005, 43, 435-479. [CrossRef]

35. Harris, J.; Zaritsky, D. The Star Formation History of the Large Magellanic Cloud. Astron. J. 2009, 139, 1243-1260. [CrossRef]

36. Paczyński, B. Evolution of single stars III. Stationary shell sources. Acta Astron. 1970, 20, 287-309.

37. Charbonnel, C. A Consistent Explanation for ${ }^{12} \mathrm{C} /{ }^{13} \mathrm{C},{ }^{7} \mathrm{Li}$ and ${ }^{3} \mathrm{He}$ Anomalies in Red Giant Stars. Astrophys. J. 1995, 453, L41. [CrossRef]

38. Charbonnel, C.; Zahn, J.-P. Thermohaline mixing: A physical mechanism governing the photospheric composition of low-mass giants. Astron. Astrophys. 2007, 467, L15. [CrossRef]

39. Iben, I., Jr. Post main sequence evolution of single stars. Annu. Rev. Astron. Astrophys. 1974, 12, 215. [CrossRef]

40. Marigo, P. Asymptotic Giant Branch evolution at varying surface C/O ratio: Effects of changes in molecular opacities. Astron. Astrophys. 2002, 387, 507-519. [CrossRef]

41. Ventura, P.; Marigo, P. Evolution and chemical yields of AGB stars: Effects of low-temperature opacities. Mon. Not. R. Astron. Soc. 2009, 399, L54-L58. [CrossRef]

42. Ventura, P.; Marigo, P. Asymptotic giant branch stars at low metallicity: The challenging interplay between the mass loss and molecular opacities. Mon. Not. R. Astron. Soc. 2010, 408, 2476-2486. [CrossRef]

43. Dell'Agli, F.; Ventura, P.; Schneider, R.; Di Criscienzo, M.; García-Hernández, D.A.; Rossi, C.; Brocato, E. Asymptotic giant branch stars in the Large Magellanic Cloud: Evolution of dust in circumstellar envelopes. Mon. Not. R. Astron. Soc. 2015, 447, 2992-3015. [CrossRef]

44. Ventura, P.; Di Criscienzo, M.; Carini, R.; D'Antona, F. Yields of AGB and SAGB models with chemistry of low- and high-metallicity globular clusters. Mon. Not. R. Astron. Soc. 2013, 431, 3642-3653. [CrossRef]

45. Scalo, J.M.; Despain, K.H.; Ulrich, R.K. Studies of evolved stars. V. Nucleosynthesis in hot-bottom convective envelopes. Astrophys. J. 1975, 196, 805. [CrossRef]

46. Bloecker, T.; Schöenberner, D. A $7 M_{\odot}$ AGB model sequence not complying with the core mass-luminosity relation. Astron. Astrophys. 1991, 244, L43-L46.

47. Renzini, A.; Voli, M. Advanced evolutionary stages of intermediate-mass stars. I. Evolution of surface compositions. Astron. Astrophys. 1981, 500, 221-239.

48. Cameron, A.G.W.; Fowler, W.A. Lithium and the s-PROCESS in Red-Giant Stars. Astrophys. J. 1991, 164, 111-114. [CrossRef]

49. Sackmann, I.J.; Boothroyd, A.I. The creation of super-rich lithium giants. Astrophys. J. Lett. 1992, 392, L71-L74. [CrossRef] 
50. Dell'Agli, F.; Hernández, D.A.; Ventura, P.; Mészáros, S.; Masseron, T.; Fernández-Trincado, J.G.; Tang, B.; Shetrone, M.; Zamora, O.; Lucatello, S. A view of the H-band light-element chemical patterns in globular clusters under the AGB selfenrichment scenario. Mon. Not. R. Astron. Soc. 2018, 475, 3098-3116. [CrossRef]

51. Mowlavi, N. Sodium production in asymptotic giant branch stars. Astron. Astrophys. 1999, 350, 73-88.

52. Boothroyd, A.I.; Sackmann, I.J. The CNO Isotopes: Deep Circulation in Red Giants and First and Second Dredge-up. Astrophys. J. 1999, 510, 232-250. [CrossRef]

53. Ventura, P. The helium contribution from massive AGBs. IAU Symp. 2010, 268, 147-152. [CrossRef]

54. Siess, L. Evolution of massive AGB stars. III. The thermally pulsing super-AGB phase. Astron. Astrophys. 2010, 512, A10-A22. [CrossRef] 